\title{
Extension of the Geometrical Two-Chain Model to high energies
}

\author{
T. Wibig \\ Experimental Physics Dept., University of Eodz, Pomorska 149/153, 90-236 Lodz, Poland
}

(February 14, 2018)

\begin{abstract}
In the paper the extension of the Geometrical Two-Chain model to higher energies is presented. The agreement with experimental data is achieved using pre-hadronization chain breakups due to coloured dipole radiation mechanism and the discretization of the soft gluon emission process.

13.85.-t, 12.39.-x, 13.87.Fh, 12.40.-y
\end{abstract}

\section{INTRODUCTION}

The Geometrical Two-Chain (G2C) model was established to describe relatively low (up to $\sqrt{s} \sim 100 \mathrm{GeV}$ ) multiparticle production processes. The model gives an unified picture of wide range of processes: from elastic hadron scattering and $\mathrm{e}^{+} \mathrm{e}^{-} \rightarrow$ hadrons reactions (Ref. [i $)$ ), through diffractive dissociation in hadron-proton interactions (Ref. [2]), to non-single-diffraction hadron-hadron (Ref. [3]) and hadron-nucleus interactions (Ref. [4]). The geometrization of the interaction picture was obtained using the eikonal function formalism in terms of hadronic matter distribution of interacting hadron (assuming point-like leptons).

The Geometrical Two-Chain model acts in two steps. The first is the soft scattering which turns incoming particles into two intermediate objects. They can be, to some extend, treated as chains concerning quarks (di-quarks) on their ends similar to the well known string structures of the LUND (Ref. [5]) or Dual Parton (Ref. [6]) models. The second stage of the interaction is the hadronization of outgoing chains.

The creation of chains (strings) and their consecutive fragmentation, in the very general sense, is a common feature of all contemporary multiparticle production process descriptions. However, the particular realization differs from model to model. In the $\mathrm{G} 2 \mathrm{C}$ model chain creation is not controlled by respective structure functions. In that sense it is rather conservative and old-fashion model but, on the other hand, such treatment has some advantages. Our knowledge of the structure function behaviour, specially at low $x$ values, is still not perfect. In our model the parametrization used does not pretend to be a fundamental law of the nature but it is just parametrization which can be changed and tuned to new data as they will appear. Generally speaking our geometrization strategy is not in contradiction to the standard structure function approach.

\section{GEOMETRICAL TWO-CHAIN MODEL}

In the $\mathrm{G} 2 \mathrm{C}$ model two chains are created on the first stage of the interaction process. The invariant mass of each of them $\left(M_{1,2}\right)$ is determined by the impact parameter $b$ of the particular collision and the CMS available energy $(\sqrt{s})$ :

$$
M_{1,2} \sim M_{0}+\left(\frac{\Omega(b)}{\Omega(0)}\right)^{\alpha}\left(\frac{\sqrt{s}}{2}-M_{0}\right),
$$

where: $M_{0}$ is a mass of the lightest hadron which can be formed from the particular quark contents of the chain and $\Omega$ is related to the mass distributions in the colliding hadrons $\left(\rho_{1,2}\right)$ by:

$$
\Omega(b)=\int \mathrm{d} \overrightarrow{\mathrm{r}} \rho_{1}(\overrightarrow{\mathrm{b}}) \rho_{2}(\overrightarrow{\mathrm{b}}-\overrightarrow{\mathrm{r}})
$$

The value of $\alpha$ parameter used in the present work is equal to 0.28 .

In the case of $\mathrm{e}^{+} \mathrm{e}^{-} \rightarrow$ hadrons reaction only one chain is created. Thus $\sqrt{s} / 2$ is replaced by $\sqrt{s}$ and the impact parameter is set to $0(\Omega(b) / \Omega(0) \equiv 1)$. One chain is created also in single diffractive case and its mass is chosen randomly from $1 / M^{2}$ distribution. Two such chains appear in double diffractive events. (In the present paper only the NSD events will be discussed.)

The hadronization starts later when all chains are formed. This is specially important for h- $\mathcal{N}$ interactions and for the high energy interactions in the multi-chain model as it will be discussed below. Hadrons are created from $q-\bar{q}$ (or diquark-antidiquark) pairs emerged from the chain CMS energy uniformly in the phase space. This is an important point of the model: no additional dynamical constrains are introduced during the hadronization phase but 
these originated from kinematical restrictions enforced by momentum and energy conservation laws. Thus the G2C model can be considered as a minimal model to study divergences between reality (experiments) and non-reducible kinematic (phase space) background. Such differences can be interpreted as introduced by some dynamics of the multiparticle production process.

The transverse momentum of created quarks originate due to tunnelling mechanism described by the formula:

$$
f\left(p_{\perp}\right) \sim \exp \left(-\frac{m_{\perp}^{2}}{\kappa}\right)
$$

and it is conserved locally. The value of $\kappa$ used in our calculations for light quark pair creation is equal to 0.28 while for the strange quarks and diquarks it is 0.38 and 0.6 respectively. Flavours of crated hadrons are given by the very few conventional suppression factors like $s / u d, q q / q$, vector/scalar. The mass difference of the hadron under consideration and the lightest hadron which can be build from particular quark configuration is also taken into account in the way similar to the one given in the Eq.(3).

The phase space density of the produced hadrons is again parametrized using the geometrical picture. The mean number of hadronization breakups of the chain $<n_{q \bar{q}}>(q-\bar{q}$ or $q q-\overline{q q}$ pairs $)$ is given by:

$$
<n_{q \bar{q}}>=n_{0}\left(\frac{\Omega(b)}{\Omega(0)}\right)^{\beta}
$$

where:

$$
n_{0}=A \ln \left(M_{\text {chain }}\right)+B \text {. }
$$

The actual number of created pairs $n_{q \bar{q}}$ is distributed due to the poissonian distribution with the respective mean value. It is directly related to the number of the first rank hadrons produced in the event. Values of $\beta, A$ and $B$ used are $0.4,6.7$ and -6.2 respectively. The correction for low $(\leq 10 \mathrm{GeV})$ chain masses is introduced. The smooth change of $A$ to the value of 3.0 at $\leq 3 \mathrm{GeV}$ ( $B$ has to be changed respectively) gives a reasonable limit for particle multiplicities at extremely low energies and can be understand, to some extent, as an effect of the resonant hadron production.

The created hadron flavours are ordered in rapidity to preserve the information of the incoming hadron quark contents. For barions the pop-corn mechanism from the LUND model (Ref. [7]) was adopted.

\section{GEOMETRICAL MULTI-CHAIN MODEL}

The G2C model as described above reproduces very well a number of interaction characteristics like e.g. mean multiplicities, multiplicity distributions, main inclusive distributions of produced particles. It has been shown in the Refs. [1,3] up to $\sqrt{s} \sim 30 \mathrm{GeV}$. For the higher energies, of course, this model has to fail. Since ISR data has been published the increase of the height of plateau in inclusive rapidity distributions (what leads to the faster $\operatorname{than} \ln (s)$ growth of the mean multiplicity) is a very well experimentally established fact. This was one of the most apparent reasons for investigations of a new physical mechanism responsible for very high energy interaction picture. In majority of up-to-date models the idea which can be called multi-string intermediate state is present. One way of introducing it is developed in DPM-like models (Ref. [6]) using multi-pomeron structures, another by using the concept of gluon emission from expanding strings is adapted to the LUND-class of models (Ref. [5, 8 ]).

In the framework of geometrical interaction picture there is a natural place for this second approach. Just after formation of two chains in the "soft scattering" phase they can be forced to break up through the "soft gluon emission" before exact hadronization starts. Such a picture is a gist of the Geometrical Multi-Chain model (GMC) which is the subject of this paper.

\section{SOFT GLUON EMISSION.}

The internal structure of the geometrical chain is in fact quite similar to the one of the LUND string. There are two coloured objects of relatively low masses each carrying invariant mass of $M_{\text {chain }}$. In the quark-antiquark (quark-diquark) rest system both coloured ends has to move with almost the velocity of light so due the existence of the colour charge the colour dipole radiation has to occur. Thus, it is expected that the soft gluon brehmsstrahlung cascade originate in such system. Its mechanism is described extensively in the ARIADNE code (Ref. [8]) adopted 
by general LUND interaction programs. The ARIADNE 4.07 is a particular realization of this idea and it was used in GMC calculations in the present paper.

The probability of the soft gluon emission is well-known (e.g. Refs. [9,5]) and can be described with analogy to QED using Sudakov form factor in the way:

$$
\frac{d P\left(p_{\perp}^{2}, y\right)}{d p_{\perp}^{2} d y}=\frac{d \sigma\left(p_{\perp}^{2}, y\right)}{d p_{\perp}^{2} d y} \exp \left(-\int_{p_{\perp}^{2}}^{p_{\perp \max }^{2}} d^{2} k \mathcal{I}\left(k^{2}\right)\right)
$$

where:

$$
\begin{gathered}
\mathcal{I}\left(k^{2}\right)=\int_{y_{\min }\left(k^{2}\right)}^{y_{\max }\left(k^{2}\right)} d y^{\prime} \frac{d \sigma\left(k^{2}, y^{\prime}\right)}{d k^{2} d y^{\prime}}, \\
d \sigma \sim \alpha_{s} \frac{d p_{\perp}^{2}}{p_{\perp}^{2}} d y .
\end{gathered}
$$

Values of the model parameter used in our GMC realization are as the default setting in the ARIADNE 4.07 program. Small difference is connected with the gluon emission suppression related to a space extension of the emission source. This works for diquark chain ends only so it is not important for the $\mathrm{e}^{+} \mathrm{e}^{-} \rightarrow$ hadrons processes. The parameter interpreted as a transverse source size is set in the present calculations to $1 \mathrm{GeV}$ while in the default ARIADNE its value is equal to $0.6 \mathrm{GeV}$. This change is related to the analysis of Bose-Einstein correlations in hadronic interactions presented in Ref. [10]. However, it is not crucial for the results of this work. Very similar results could also be obtained with the value of $0.6 \mathrm{GeV}$ and respective slight change of other model parameters.

The important difference of the GMC model in comparison with standard ARIADNE soft gluon emission is in the implementation of the discretization of the process. The idea is described in the Ref. [11]. In this paper the (logarithmic) phase space $\left(\ln \left(\mathrm{p}_{\perp}^{2}\right), \mathrm{y}\right)$ is divided into discrete cells and the point is that each cell can be occupied by only one brehmsstrahlung gluon. The theoretical basis of this picture is widely discussed in the original paper. In the most general way it can be expressed as following: if there are two gluons occasionally emitted too close one to the other they combine together conduced finally to one "effective gluon". The measure of the closeness can be derived, to some extend, from the running coupling constant QCD and for two gluons the minimum distance in rapidity should be of order of $11 / 6$ (Ref. [11).

In our model this idea is adopted in the way that gluons are produced by the standard ARIADNE coloured antenna and then the gluon recombination initiates. The distance between gluons is defined in three-dimensional $\left(\ln \left(\mathrm{p}_{\mathrm{x}}^{2}\right)\right.$, $\left.\ln \left(\mathrm{p}_{\mathrm{y}}^{2}\right), \mathrm{y}\right)$ space. The recombination procedure starts with the pair of gluons closest each other and finished when all "effective gluons" stay apart by at least the value of a parameter denoted by $\delta \mathrm{y}_{\mathrm{g}}$ which is going to be adjusted to the data.

After soft gluon emissions the main chain is broken into smaller parts. In the present version of the GMC model each gluon is turned to $q \bar{q}$ pair and its momentum is shared between them by halves.

Further, chain pieces (each one containing again $q-\bar{q}(q q-q)$ pair) of smaller masses and with some additional transverse momenta hadronize according to the $\mathrm{G} 2 \mathrm{C}$ model described above.

\section{RESULTS AND DISCUSSION}

If the mean particle multiplicity produced during the hadronization phase is proportional to the logarithm of the chain mass then the emission of gluons with non-zero transverse masses and consecutive breakup of the chain before hadronization leads to the increase of the average multiplicity. Also the additional transverse momentum originated from the soft gluon brehmsstrahlung should increase the mean secondary hadron $\mathrm{p}_{\perp}$. The question is: is it possible to reach the quantitative agreement with measurements preserving the idea described above with acceptable parameter values?

As it was done in Ref. [1] first we proceed with the mean hadron multiplicities in $\mathrm{e}^{+} \mathrm{e}^{-} \rightarrow$ hadrons reactions. The geometrical model parameters $\alpha$ and $\beta$ in Eqs.(11) and (4) do not interfere this $(\Omega \equiv \Omega(0))$. The same can be said about parameters of the suppression of short wave length gluon emissions by extended (diquark) chain ends in the ARIADNE model as described in Ref. [8]. Values of $A$ and $B$ in Eq.(5) were first obtained at this point. The parameter $\delta \mathrm{y}_{\mathrm{g}}$ of the discrete QCD approximation used has a small influence on the $\mathrm{e}^{+} \mathrm{e}^{-}$mean multiplicity data due to relatively small range of chain masses under study (the results for $\delta \mathrm{y}_{\mathrm{g}}=0$ are shown in the Fig. 11 by the thin solid line). The result of the GMC model calculations is presented in the Fig.1] by the thick solid line. It was obtained with 
the $\delta y_{\mathrm{g}}=1.45$ adjusted using mainly other data what will be shown below. Results of calculations without the soft gluon emission (pure G2C model with the same $A$ and $B$ parameter values) are given by the dashed line. Expected enhancement is clearly seen and the accuracy achieved is very satisfactory.

Predictions of the LUND interaction model (ARIADNE + JETSET with the set of parameters adjusted to the DELPHI data) are given also by the short dashed line. At this point it should be clarified why the LUND model leads to the very similar results as ours which uses also ARIADNE procedures to describe soft gluon emissions but with the discretization procedure hereafter. There are of course differences in the hadronization description but they are not essential here. The important difference is in the treatment of coloured antenna radiated gluons. The discretization process used in our GMC model works on a gluon level. Finally each gluon ("effective gluon") has to form a common end of two chains which then fragment independently. Thus each soft emission creates one additional chain which is forced to hadronize to at least one final hadron. In the LUND picture the low mass (below some critical value) jets are combined with the nearby ones before they hadronize. Final results, as it can be seen, are similar but the underlying physics is quite different.

In the next step parameters $\alpha$ and $\beta$ in Eqs. (11) and (4) has to be adjusted using the proton-proton multiplicity data.

The mean charged multiplicity, multiplicity moments (defined as $\mathrm{C}_{\mathrm{k}}=\left\langle\mathrm{n}_{\mathrm{ch}}^{\mathrm{k}}\right\rangle /\left\langle\mathrm{n}_{\mathrm{ch}}\right\rangle^{\mathrm{k}}$ ) data and the GMC model results are presented in the Figs.2 and 3 . The result of G2C calculations is also given.

To see the influence of the discretization of the gluon brehmsstrahlung process result of the calculation with the $\delta y_{g}$ parameter equal to 0 is given in the Fig. 1 and 1 by the thin solid line. The difference is not very large but it has to be pointed out here that the number of gluons emitted primarily is limited by the threshold value of $\mathrm{p}_{\perp}$ of emitted gluons used in coloured dipole radiation mechanism adopted in ARIADNE code. Its value was set to $0.6 \mathrm{GeV} / \mathrm{c}$. In principle, such limit is equivalent to the assumption that all softer gluons are effectively included in harder emissions or hadronization processes. Thus $\delta \mathrm{y}_{\mathrm{g}}=0$ does not mean absence clustering of emitted gluons, but only an omission of joining a relatively "massive" gluons.

The results of the LUND model calculations with the FRITIOF (Ref. [12]) program are also presented in the Fig.2 by the short dashed line. For the soft gluon emission there are few different sets of ARIADNE program parameters. Here the same set called "DELPHI" was used as for the description of $\mathrm{e}^{+} \mathrm{e}^{-} \rightarrow$ hadrons mean multiplicities in the Fig.1.

As it can be seen mean multiplicities are reproduced by GMC model very well while obtained multiplicity distributions are slightly narrower than these observed in experiments at the highest energies (Fig.33). However, the difference is not very significant and, what is more important, the tendency of decreasing the higher moments with the increasing interaction energy seen for G2C converts toward experimental results.

Obtained values of the model parameters $\alpha, \beta, A$ and $B$ parameters were then used to determine finally the value of $\delta \mathrm{y}_{\mathrm{g}}$ using the data on secondary hadron transverse momenta.

The dependence of the averaged transverse momentum on energy is shown in the Fig. A. The constant value (for high energies) predicted by the G2C model is settled by the value of the $\kappa$ parameter in the Eq.(3). The same value of $\kappa$ with the soft gluon radiation mechanism gives an increase of average $\mathrm{p}_{\perp}$ in perfect agreement up to the highest available energy accelerator data. The average transverse momenta of produced particles are quite sensitive to the gluon clustering mechanism. Thus they were used to determine the value of $\delta \mathrm{y}_{\mathrm{g}}$ in the GMC model. For example the very strong increase of the average value of $\mathrm{p}_{\perp}$ for $\delta \mathrm{y}_{\mathrm{g}}=0$ is shown in the Fig. 1 (thin solid line). Finally $\delta \mathrm{y}_{\mathrm{g}}$ was found to be equal to 1.45 .

The most significant and crucial test of the GMC model is the comparison with inclusive energy ( longitudinal momentum or (pseudo)rapidity ) data. The increase of the plateau and the breaking of the Feynman scaling at high energies are two features of the highest importance in applications of the model for the hadronic cascade in the thick media.

Rapidity distributions for SPS and Tevatron energies are presented in Fig. 5. The very significant change from G2C to GMC model predictions is seen and the agreement obtained with the GMC model is meaningful. The results of FRITIOF with DELPHI set of parameters for ARIADNE are also given for comparison.

However good the agreement is, it can not be treated as a proof of the correctness of the proposed model. There are other very well-known model available consistent with data as well. Two main classes of such models are: the one based on DPM picture (There are many particular realizations of the DPM idea see e.g. Ref. [6]) and the second - relativistic string model of LUND (Ref. [j]). There are many particular realization of the DPM idea. The most complete comparison between some of them and the data is given in the Ref. [13. The very interesting model calculations are presented in the Ref. [14]. The multi-pomeron exchange concept is combine there with the impact parameter description and the standard LUND string fragmentation procedures JETSET are used. The LUND model itself for proton-proton and $\mathrm{e}^{+} \mathrm{e}^{-} \rightarrow$ hadrons reactions is available as a package of FRITIOF, ARIADNE, PYTHIA and JETSET routines. Results obtained using these codes are presented for all discussed interaction characteristics in the Figures. It should be said here that even if the particular feature is better described by one model it is not an 
argument against or for any model. The LUND interaction picture is of course much more complete what was shown in a number of papers. The point is that our GMC model with its physical assumption is also able to reproduce the data quite well.

\section{SUMMARY}

The extension of the Geometrical Two-Chain multiparticle production mechanism to the higher energies is obtained by the introduction of the soft gluon emission process. The framework of the ARIADNE coloured antenna radiation with the recent idea of discretization of the process is used to perform high energy chain pre-hadronization breakups. The default ARIADNE 4.07 parameter values were used. The discrete net step $\delta \mathrm{y}_{\mathrm{g}}$ was found to be equal to 1.45 which is close to the presumed value of $11 / 6$. The difference is not significant according to the slightly different implementation of the $\left(\ln \left(\mathrm{p}_{\perp}^{2}\right), \mathrm{y}\right)$ cell idea.

The consistency between multiparticle hadronic interactions data and predictions of the GMC model presented in this paper and the solid theoretical basis of the model grant a capability of reasonable extrapolation of the model to high energies.

[1] T. Wibig and D. Sobczyńska, Phys. Rev. D49, 2268 (1994).

[2] D. Sobczyńska and T. Wibig , Bull. Russ. Acad. Sci. 58, 1970 (1994).

[3] T. Wibig and D. Sobczyńska, Phys. Rev. D50, 5657 (1994).

[4] T. Wibig and D. Sobczyńska, J. Phys. G21, 29 (1995).

[5] B. Anderson, G. Gustafson G. Ingelman and T. Sjöstrand, Phys. Rep. 97, 31 (1983); H. Bengtsson and T. Sjöstrand, Comput. Phys. Commun. 46, 43 (1987).

[6] P. Aurenche, F. W. Bopp, A. Capella, J. Kwieciński, M. Maire, J. Ranft and J. Tran Thanh Van, Phys. Rev. D45, 92 (1992); K. Werner, Phys. Rep. 232, 87 (1993); A. Capella, U. Sukhatme, C-I Tan and J. Tran Thanh Van, Phys. Rep. 236, 225 (1994); R. S. Fletcher, T. K. Gaisser, P. Lipari and T. Stanev, Phys. Rev. D50, 5710 (1995); J. Ranft, Phys. Rev. D51, 64 (1995).

[7] B. Anderson, G. Gustafson and T. Sjöstrand, Phys. Scripta 32, 574 (1985).

[8] L. Lönnblad, Comput. Phus. Commun. 71, 15 (1992).

[9] G. Gustafson, Phys. Lett. B175, 453 (1986); G. Gustafson and U. Pettersson, Nucl. Phys. B306, 746 (1988); B. Andersson et al., Z. Phys. C43, 625 (1989); B. Andersson, G. Gustafson and L. Lönnblad, Nucl. Phys. B339, 393 (1990).

[10] T. Wibig, Phys. Rev. 54, 707 (1995).

[11] B. Andersson, G. Gustafson and J. Samuelsson, Nucl. Phys. B463, 217 (1996).

[12] B. Andersson, G. Gustafson and Hong Pi, Z. Phys. BC57, 485 (1993).

[13] J. Knapp, D. Heck and G. Schatz, Forschungszentrum Karlsruhe Report FZKA-5828, Karlsruhe (1996).

[14] T. Sjöstrand and M. van Zijl, Phys. Rev. D36, 2019 (1987).

[15] G. Giacomelli, Nucl. Phys. B25, 30, (1992); M. Schmelling, Phys. Scripta 51, 683 (1995).

[16] J. Whitemore, Phys. Rep. 10, 273 (1974); U. Amaldi and K. R. Schubert, Nucl. Phys. B166, 301 (1980); G. J. Alner et al., Phys. Lett. 138B, 304 (1984); 160B, 199 (1985); 167B, 476 (1986); Phys. Rep., 154, 247 (1986); Z. Phys. C 33, 1 (1986); R. E. Ansorge et al., Z. Phys. C 43, 357 (1989); F. Abe et al., Phys. Rev. D 41, 2330 (1990).

[17] F. Abe et al., Phys. Rev. Lett. 61, 1819 (1988). 


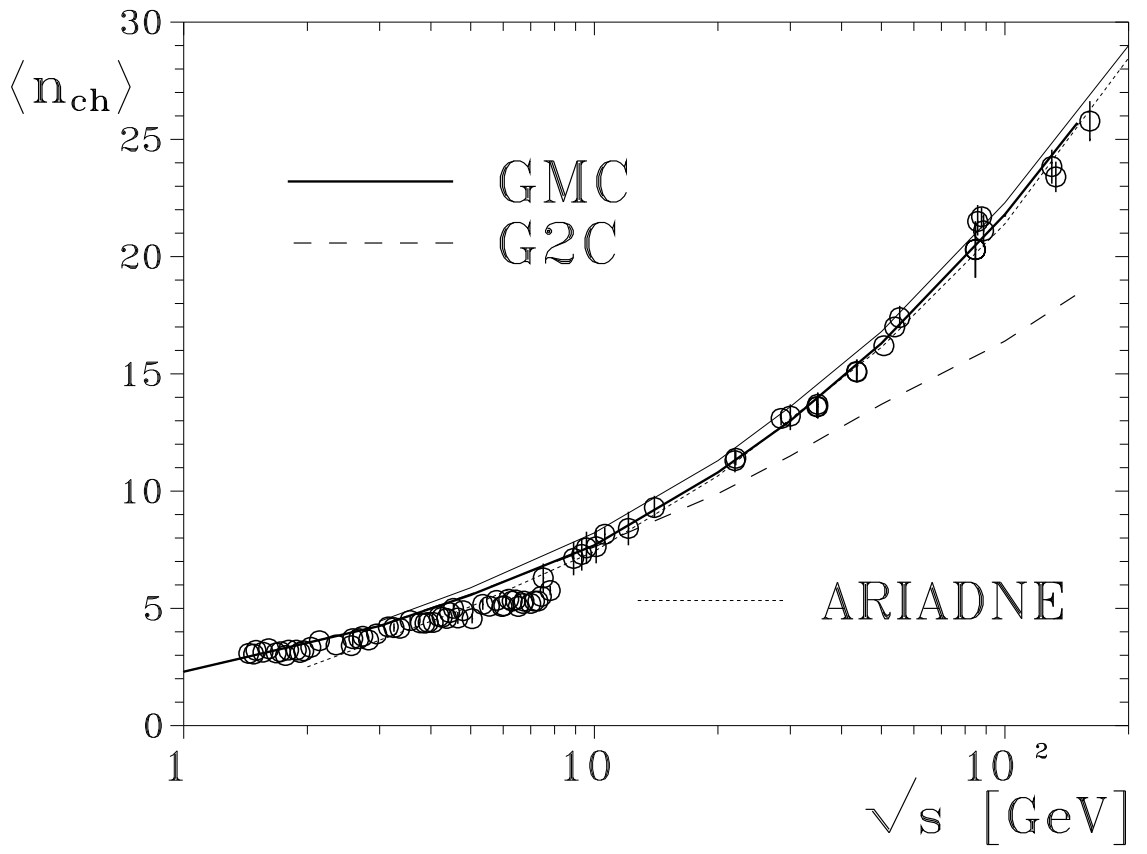

FIG. 1. Mean charged multiplicity in $\mathrm{e}^{+} \mathrm{e}^{-} \rightarrow$ hadrons reactions. The thick solid line represents result of the Geometrical Multi-Chain model calculations while the long dashed one is for the same parameter value set but without soft gluon emission process (pure two-chain picture). Thin solid line represents the GMC model results with weaker gluon clustering $\left(\delta \mathrm{y}_{\mathrm{g}}=0\right)$. The short dashed line is a result of the LUND interaction model calculations. The data point are from [15].

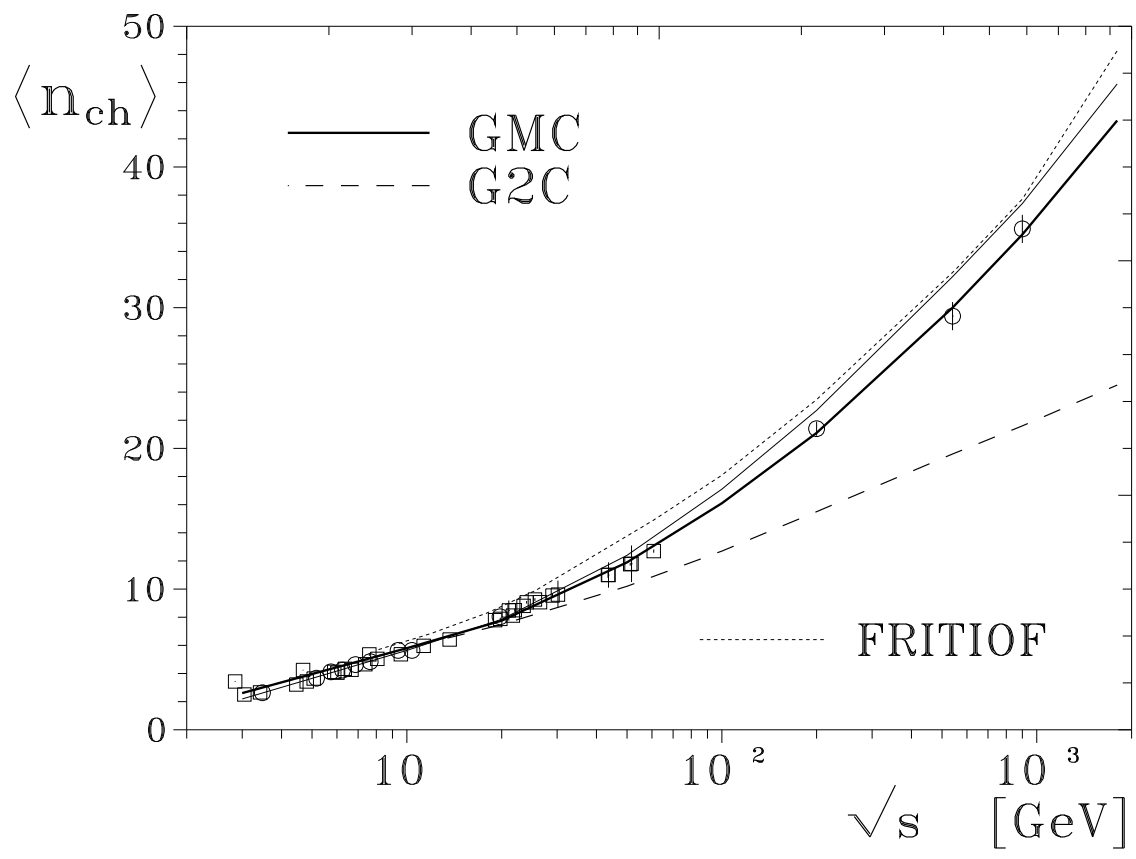

FIG. 2. Mean charged multiplicity in hadron-proton multiparticle production reactions. The thick solid line represents result of the Geometrical Multi-Chain (GMC) model calculations while the long dashed one is for the pure two-chain (G2C) interaction picture. Thin solid line represents the GMC model results with weaker gluon clustering $\left(\delta \mathrm{y}_{\mathrm{g}}=0\right)$. The short dashed line is a result of the LUND model calculations. The data point are from [16]. 


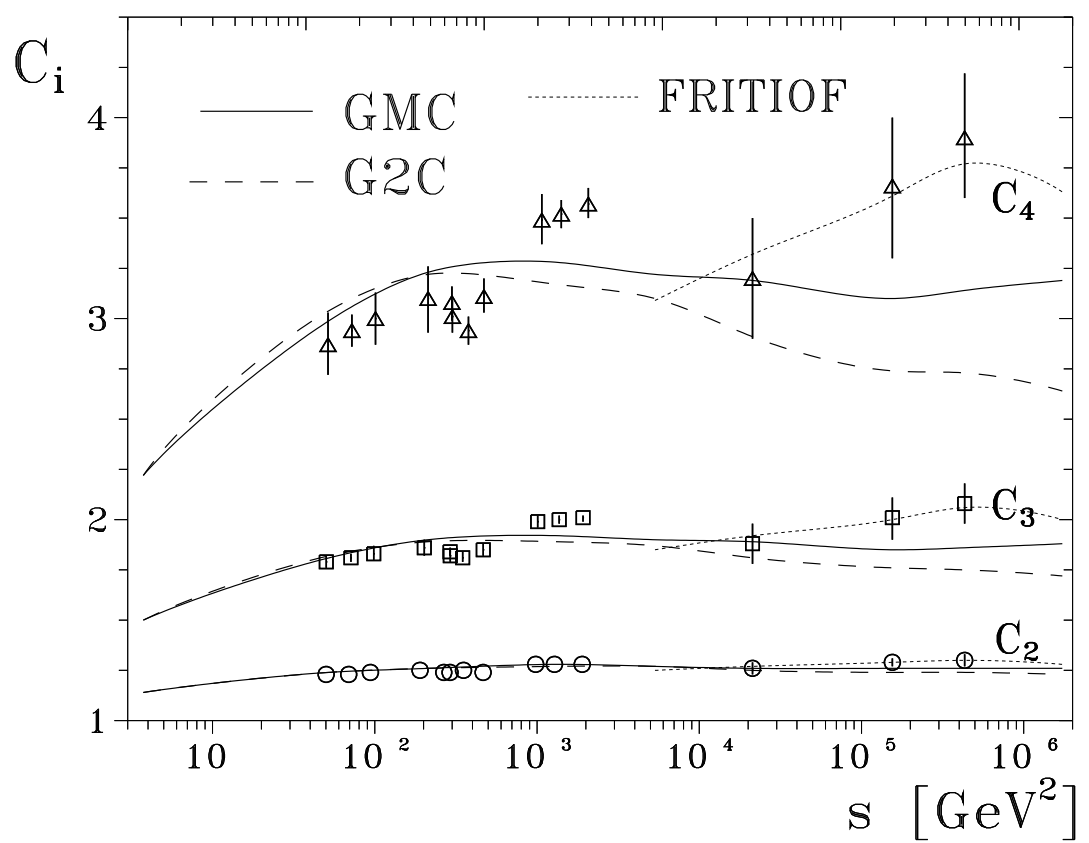

FIG. 3. Normalized charged multiplicity distribution moments for hadron- proton multiparticle production reactions. The solid line represents result of the Geometrical Multi-Chain (GMC) model calculations while the long dashed one is for the pure two-chain (G2C) interaction picture. The short dashed line is a result of the LUND model calculations. The data point are from [16].

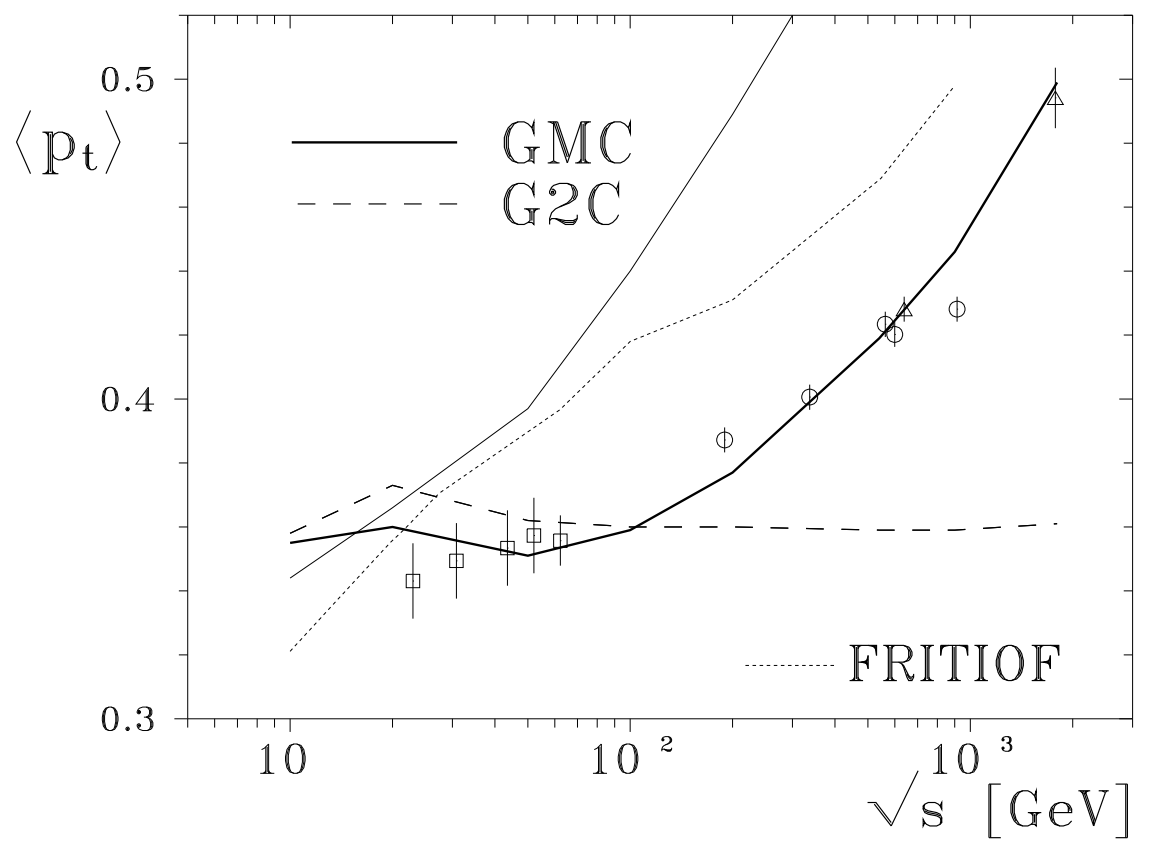

FIG. 4. Mean charged particle transverse momentum for hadron-proton multiparticle production reactions. The line description as in the Fig. 2. The data point are from [17]. 


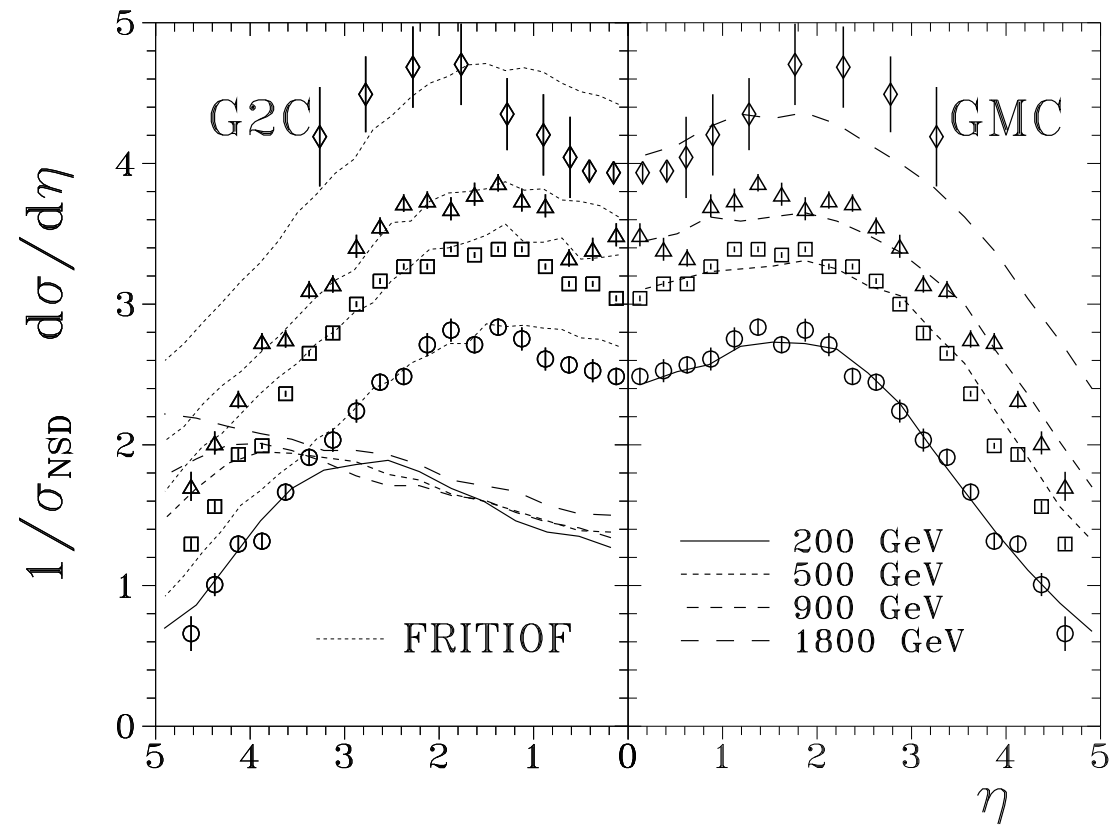

FIG. 5. Inclusive rapidity spectra of charged particles in proton-proton interactions at SPS and Tevatron energies. The results for $\mathrm{G} 2 \mathrm{C}$ model are given in the left part of the Figure while the present GMC model calculations are in the right part. The short dashed lines in the left part are results of the LUND model. The data point are from [16]. 